Puente-Maxera, F.; Mahedero-Navarrete, M.P.; Méndez-Giménez, A.; Martínez de Ojeda, D. (2020) Sport Education, Roles and Vulnerability. Influence on Responsibility and Intercultural Competence. Revista Internacional de Medicina y Ciencias de la Actividad Física y el Deporte vol. 20 (80) pp. 487-503 Http://cdeporte.rediris.es/revista/revista80/arteducacion1185.htm

DOI: https://doi.org/10.15366/rimcafd2020.80.001

\title{
ORIGINAL
}

\section{EDUCACIÓN DEPORTIVA, ROLES Y VULNERABILIDAD. INFLUENCIA EN LA RESPONSABILIDAD E INTERCULTURALIDAD}

\section{SPORT EDUCATION, ROLES AND VULNERABILITY. INFLUENCE ON RESPONSIBILITY AND INTERCULTURAL COMPETENCE}

\author{
Puente-Maxera, F.1; Mahedero-Navarrete, M. P. ${ }^{2}$; Méndez-Giménez, A. ${ }^{3}$; \\ Martínez de Ojeda, D. ${ }^{4}$
}

1 Doctorando en Educación y Psicología. Facultad de Formación del Profesorado y Educación, Departamento de Ciencias de la Educación. Universidad de Oviedo (España) fedepuentem@hotmail.com

${ }^{2}$ Doctora en Ciencias de la Actividad Física y del Deporte. Consejería de Educación, Junta de Andalucía (España) pilarmahedero@hotmail.com

${ }^{3}$ Doctor en Ciencias de la Actividad Física y del Deporte. Facultad de Formación del Profesorado y Educación, Departamento de Ciencias de la Educación. Universidad de Oviedo (España) mendezantonio@uniovi.es

${ }^{4}$ Doctor en Ciencias de la Actividad Física y del Deporte. Consejería de Educación, Región de Murcia (España) diegomop@yahoo.es

Código UNESCO / UNESCO code: 5899 Educación Física y Deporte / Physical Education and Sport.

Clasificación Consejo de Europa / Council of Europe classification: 4. Educación Física y Deporte comparado / Compared Sport and Physical Education; 5. Didáctica y metodología / Didactics and methodology.

Recibido 11 de octubre de 2018 Received October 11, 2018

Aceptado 2 de febrero de 2019 Accepted February 2, 2019

\section{RESUMEN}

El presente estudio examinó los efectos de dos dinámicas de roles (fija y rotativa) sobre los niveles de responsabilidad y competencia intercultural en adolescentes españoles de un contexto socialmente vulnerable. Formaron parte del estudio 96 estudiantes de primer curso de educación secundaria $\left(M_{\mathrm{edad}}=12.2\right)$ de un centro ubicado en Andalucía (España). Siguiendo un diseño cruzado simple, cuasiexperimental y con medidas repetidas (pretest-post1-post2), los participantes asistieron a dos unidades didácticas consecutivas con Educación Deportiva 
sobre baloncesto y floorball, respectivamente. Se recabó información mediante entrevistas semi-estructuradas y cuestionarios. Se evidenció un mayor impacto de los roles fijos en la responsabilidad social y en las variables interculturales (sensibilidad y comportamiento), con especial incidencia en varones. Ligeras consecuencias negativas fueron halladas en los niveles de responsabilidad de estudiantes de sexo femenino. Se desvela la importancia de asumir roles fijos en contextos socialmente vulnerables, así como en experiencias iniciales con Educación Deportiva.

PALABRAS CLAVE: educación física, modelos pedagógicos, responsabilidad, estudiantes en riesgo.

\section{ABSTRACT}

This paper examines the effect of two different roles' strategies (permanent and rotating) on Spanish adolescents' responsibility and intercultural competence levels. Following a quasi-experimental, simple crossover design with repeated measures (pretest-post1-post2), 96 high school students $\left(M_{\text {age }}=12.2\right)$ and one teacher participated part in two consecutive Sport Education-based teaching units of basketball and floorball, respectively. Data was collected through semistructured interviews and questionnaires. Permanent roles were associated with improvements on social responsibility and intercultural variables (sensibility and behavior), especially in males. There are slightly negative consequences on female' responsibility levels. Findings reveal and emphasize the importance of using permanent roles when dealing with socially vulnerable contexts, as well as first experiences with Sport Education.

KEY WORDS: physical education, pedagogical models, responsibility, at risk students.

\section{INTRODUCCIÓN}

La relación entre las condiciones de vida del alumnado y sus posibilidades de aprendizaje ha sido objeto de estudio durante décadas (Serra, y Canciano, 2006). En el marco de dicha producción, especial interés se ha vertido sobre la población residente en contextos de vulnerabilidad social, entendidos estos como aquellos donde conviven personas que guardan una relación distorsionada con las instituciones (familia, escuela, sanidad, etc.), caracterizada por la acumulación de experiencias negativas, sentimientos de incompetencia y bajas expectativas sociales (Haudenhuyse, Theeboom, y Nols, 2012). A nivel educativo, muchas de las necesidades que emanan de estos contextos han encontrado respuesta en la innovación. Entre las distintas tipologías de innovación existentes (Rivas, 2002), aquella concerniente a los procedimientos didácticos ha cobrado especial relevancia en los últimos años. Desde el área de Educación Física (EF), fruto de dichos procesos de investigación e innovación, han surgido varias propuestas que han guiado la acción docente, dando lugar a modelos pedagógicos (Casey, y Dyson, 2009; Hastie, y Casey, 2014; Metzler, 2011). 
Metzler (2011) establece un total de ocho modelos pedagógicos. Uno de estos, la Educación Deportiva (Siedentop, 1994), nace en respuesta a la verticalidad de la instrucción directa, pretendiendo brindar experiencias auténticas, significativas y contextualizadas a todo el alumnado (Siedentop, Hastie, y van der Mars, 2011). Entre sus objetivos fundamentales, reside el deseo de promover jugadores competentes, cultos y entusiastas (Siedentop et al., 2011). El modelo viene caracterizado por seis aspectos básicos: afiliación, temporada, competición formal, registro de datos, festividad, y evento final.

Varias investigaciones han sido desarrolladas bajo los postulados de esta metodología (ver Araújo, Mesquita, y Hastie, 2014; Hastie, Martínez de Ojeda, y Calderón, 2011). Los hallazgos más sobresalientes destacan las bondades del modelo en la promoción de valores prosociales (García-López, y Gutiérrez, 2013), en los aspectos motivacionales (Méndez-Giménez, Fernández-Río, y Méndez-Alonso, 2015), o el nivel de competencia (Hastie, Sinelnikov, y Guarino, 2009), entre otros. No obstante, pocas han sido las propuestas dirigidas a examinar la influencia de este modelo sobre la responsabilidad. Destacan los estudios de Menéndez-Santurio y Fernández-Río $(2016$; 2017) con adolescentes españoles, a partir de propuestas híbridas e incluyendo el kickboxing como contenido principal. Los autores constataron el impacto positivo de sus intervenciones sobre la responsabilidad personal y social del alumnado. Es igualmente reseñable el trabajo de Fernández-Río, Méndez-Giménez, y MéndezAlonso (2017), quienes encontraron mejoras significativas en la responsabilidad social de estudiantes adolescentes, destacando la importancia de la asunción de roles sobre el desarrollo de dicha variable.

Otro factor frecuentemente identificado como decisivo en la configuración de contextos de vulnerabilidad social es la presencia de alumnado culturalmente diferente (Subirats, Carmona, y Torruella, 2005). En las últimas décadas, una amplia producción realizada desde distintas áreas disciplinares se ha dirigido a explorar, conocer y mejorar los procesos de enseñanza-aprendizaje en escenarios multiculturales. La EF no ha sido ajena a dicha producción, siendo varios los autores que comulgan con la existencia de una EF intercultural (Contreras, Gil, Cecchini, y García-López, 2007). Así, destacan trabajos dirigidos tanto a alumnado (Grimminger-Seidensticker, y Möhwald, 2017) como profesorado (Pastor-Vicedo, Contreras, Gil-Madrona, y Cuevas-Campos, 2016). Sin embargo, son escasas las intervenciones en espacios multiculturales diseñadas a partir de modelos pedagógicos en general, y con Educación Deportiva en particular (Méndez-Giménez, Puente-Maxera, y Martínez de Ojeda, 2017).

Numerosas investigaciones han ido orientadas a examinar el potencial de los elementos configuradores de la Educación Deportiva, asumidos estos como "innegociables" (Hastie, y Casey, 2014). Hastie, Ward y Brock (2017) examinaron los efectos de los agrupamientos en función de los niveles de habilidad del alumnado, evidenciando mejoras en el juego de estudiantes de nivel bajo cuando se enfrentaban a otros de nivel similar. Dichas mejoras fueron superiores a las reportadas por quienes actuaron en agrupaciones mixtas. Otro aspecto inherente al modelo son las responsabilidades (Siedentop, 1994) pudiendo ser de carácter 
fijo o rotativo. Así, dos estudios recientes han explorado el impacto de las dinámicas de roles (fija y rotativa) sobre los aspectos motivacionales (PuenteMaxera, Méndez-Giménez, y Martínez de Ojeda, 2018b) e interculturales (Puente-Maxera, Méndez-Giménez, y Martínez de Ojeda, 2017) del alumnado preadolescente. El primero advierte mejoras derivadas de ambos tratamientos (roles fijos y rotativos) en cada una de las necesidades psicológicas básicas, así como en la orientación a la tarea, siendo la dinámica rotativa la única en promover mejoras significativas en el clima orientado al ego. Igualmente, el segundo estudio (Puente-Maxera et al., 2017) encuentra mejoras en la sensibilidad intercultural del alumnado derivadas tanto de la rotación de roles como de la dinámica de roles fijos, siendo superiores las provocadas por la primera.

Más allá de estas aportaciones, en la actualidad no constan estudios que hayan examinado el efecto de las dinámicas de roles sobre los niveles de responsabilidad, siendo extensible estas carencias a contextos de educación secundaria. El presente estudio, a partir de un diseño cruzado simple, pretende superar estas limitaciones y comprobar el impacto de las dinámicas de roles (fija y rotativa) sobre los niveles de responsabilidad y de competencia intercultural en alumnado adolescente. Se establece como primera hipótesis que (a) la rotación de roles supondría mejoras significativas en los niveles de responsabilidad personal y social, mayores que las provocadas por la dinámica fija (Hipótesis 1); en segundo lugar, (b) los roles rotativos provocarían aumentos significativos en cada una de las dimensiones positivas del comportamiento intercultural, así como un descenso significativo en la irresponsabilidad, siendo estos resultados superiores a los suscitados por la dinámica de roles fijos (Hipótesis 2); y en último lugar, (c) la dinámica rotativa promovería mejoras significativas en la sensibilidad intercultural del alumnado mayores que las provocadas por los roles fijos (Hipótesis 3).

\section{MATERIAL Y MÉTODO}

\section{Diseño}

Se sigue un diseño cruzado simple, cuasi-experimental y con medidas repetidas (figura 1). Estudios previos sobre el modelo de Educación Deportiva han sido abordados mediante diseños cuasi-experimentales (Martínez de Ojeda, MéndezGiménez, y Valverde, 2016) y diseños cruzados (Calderón, Martínez de Ojeda, y Hastie, 2013). Se emplean instrumentos tanto cuantitativos (p.ej., cuestionarios) como cualitativos (p.ej., entrevistas). A efectos de garantizar la fidelidad de la intervención, se tomaron en cuenta las recomendaciones de Hastie y Casey (2014) para estudios basados en modelos de enseñanza, que priorizan tres aspectos: (a) una profunda descripción de los elementos curriculares de las unidades, (b) un proceso detallado sobre la validación del modelo pedagógico y (c) una rica descripción del contexto. El diseño fue supervisado por dos doctores (expertos en modelos de enseñanza). 
Participaron 96 estudiantes (53 chicos y 43 chicas) con edades entre los 11 y 15 años $(M=12,2 ; D T=0,82)$ adscritos a cinco grupos naturales de primero de educación secundaria ubicados en un centro en el sur de España. A nivel étnicocultural, seis estudiantes eran de origen inmigrante (principalmente, magrebíes) y 90 de origen autóctono (nacionalidad española). De estos últimos, seis estudiantes eran de etnia gitana. También cabe destacar la presencia de un 13\% de estudiantes residentes en un barrio marginado. El alumnado carecía de experiencia con Educación Deportiva. Por su parte, la docencia fue asumida por una profesora de EF (doctora en Ciencias de la Actividad Física) con 11 años de experiencia docente ( 7 de ellos aplicando Educación Deportiva). Se obtuvo el consentimiento informado tanto de las familias y tutores legales del alumnado como del equipo directivo y el departamento de EF del centro. A nivel ético, la intervención contó con el respaldo de la comisión de doctorado de una universidad ubicada en el norte de España.

\section{Unidades didácticas implementadas}

Siguiendo los postulados de Educación Deportiva, se diseñaron dos unidades didácticas (baloncesto y floorball) de doce sesiones de 60 minutos cada una (dos veces por semana). Ambas propuestas progresaron por las siguientes fases: (i) introductoria, (ii) dirigida, (iii) práctica autónoma, (iv) competición formal y (v) evento final. Cada grupo-clase fue organizado en equipos de cinco o seis miembros, establecidos por la docente, siguiendo criterios de género (chico/chica), nacionalidad (autóctono/inmigrante) y etnia (alumnado de etnia gitana). En el proceder de cada grupo, los estudiantes ejercieron distintos roles: entrenador, preparador físico, responsable de material y mediador en las situaciones de conflicto. A estas responsabilidades, cabe añadir la labor arbitral (duty team), de carácter rotativo, y asumida por cada uno de los participantes.

Cada unidad de enseñanza fue abordada en todos los grupos-clase de forma simultánea, de modo que, en primer lugar, se llevó a cabo la unidad de baloncesto (octubre-diciembre) y, seguidamente, la de floorball (enero-marzo). El tratamiento diferencial (variable independiente) estribó en la dinámica de roles asumida en cada unidad, pudiendo ser (a) fija o (b) rotativa. De esta forma, cuando unos grupos asumían roles fijos, los otros trabajaban a partir de roles rotativos y, al dar paso a la segunda unidad, los grupos alternaban el tratamiento. Siguiendo los intereses del estudio, los grupos A y B $(n=43)$ fueron emparejados pasando a ser denominados Grupo Experimental 1 (GE-1, en adelante), mientras que en los grupos C, D y E $(n=53)$ se hizo lo propio, resultando en el Grupo Experimental 2 (GE-2, en adelante). El GE-1 comenzó con roles rotativos, mientras que el GE-2, con roles fijos. Respecto a la rotación de roles, fue efectuada cada dos clases. Para ello, los equipos disponían de una planilla en la que se establecía el orden cronológico (sesiones) seguido en cada uno de los roles. 


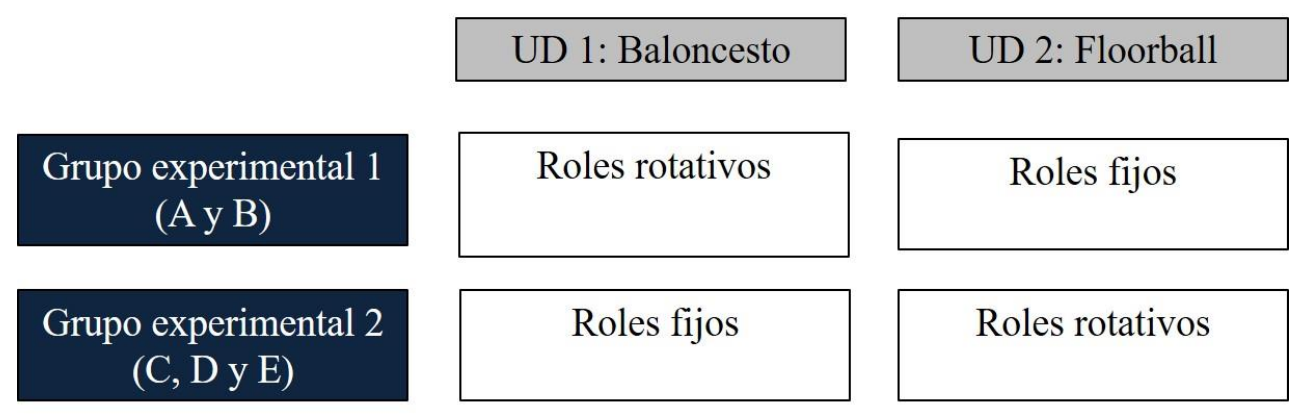

Figura 1. Diseño cruzado del estudio

\section{Instrumentos}

\section{Percepción del alumnado}

\section{Cuestionarios}

Responsabilidad personal y social. Se utilizó la adaptación al castellano del Personal and Social Responsibility Questionnaire (Escartí, Gutiérrez y Pascual, 2011), compuesto por 14 ítems valorados en una escala Likert de 1 (totalmente en desacuerdo) a 6 (totalmente de acuerdo) y divididos en dos factores: responsabilidad personal (p.ej.. "Trato de esforzarme aunque no me guste la tarea") y responsabilidad social (p.ej.., "Respeto a los demás").

Comportamiento intercultural. Se empleó la Student's Behaviors' Self-evaluation Scale (SBSS; Kellis, Vernadakis, Albanidis, Derri y Kourtesses, 2010) que mide el comportamiento social del alumnado en contextos multiculturales. Consta de 18 ítems distribuidos en cinco escalas: Relación ( 3 ítems), Irresponsabilidad (3 ítems), Recompensa (4 ítems), Ayuda (4 ítems) y Metas (4 ítems). Para el presente estudio, fue omitida la escala metas al considerarla irrelevante para los intereses del estudio, al igual que lo realizado en el contexto español en estudios previos con Educación Deportiva (Méndez-Giménez et al., 2017). Las preguntas vienen valoradas en una escala Likert que va de 1 (nunca) a 5 (siempre).

Sensibilidad Intercultural. Se administró la Escala de Sensibilidad Intercultural (Sanhueza y Cardona, 2008) compuesta por diez ítems cuyas respuestas oscilan entre 1 (nunca es cierto) y 4 (siempre es cierto). El instrumento presenta una estructura bifactorial: (a) respuesta emocional positiva (REP, 7 ítems) y (b) respuesta emocional negativa (REN, 3 ítems). Dados los pocos ítems en la REN, para el presente estudio dicha sub-escala fue adaptada, viéndose incrementada en dos reactivos.

Los cuestionarios fueron administrados mediante la plataforma GoogleForm en horario de clase, en el aula de informática del centro. Los estudiantes invirtieron un tiempo aproximado de 20 minutos en completarlos. 
Entrevistas. La profesora a cargo de impartir docencia fue objeto de tres entrevistas individuales semi-estructuradas en diferentes momentos (antes -E1, después de la primera unidad -E2-, y después de la segunda -E3-). Estas fueron conducidas por uno de los investigadores, siendo revisadas previamente por dos expertos ajenos a la recogida de datos. Tal como afirma Ruíz (2012), la entrevista semi-estructurada pretende comprender, más que explicar los hechos y carece de un esquema fijo de categorías de respuesta. Cada entrevista tuvo una duración media aproximada de 30 minutos y fue desarrollada mediante la herramienta Skype.

\section{Análisis de datos cuantitativos}

La consistencia interna se examinó mediante el alfa de Cronbach de cada una de las variables y sus escalas. Los resultados informan de niveles de aceptabilidad ( $\alpha>0,70$, Nunnally, 1978) en todos los factores, a excepción de la Responsabilidad Personal, la Irresponsabilidad (PreT) y la REN (PreT y Post1). No obstante, considerando el interés que suponían para el estudio, y según lo realizado en estudios previos (Spittle y Byrne, 2009), se utilizaron aquellos valores situados entre 0,60 y 0,70 . Los resultados en el Post1 en la REN $(\alpha=0,51)$ fueron ignorados para análisis subsiguientes.

La normalidad de las variables fue revisada mediante el test de KolmogorovSmirnov y la homogeneidad de varianzas mediante la prueba de Levene. Los resultados informaron que no se cumplía con el criterio de normalidad por lo que se procedió al empleo de pruebas no paramétricas. Se calcularon los estadísticos descriptivos (medias y desviaciones típicas) y se ejecutó la prueba de rangos de Wilcoxon para dos muestras relacionadas, a efectos de comparar resultados en los diferentes momentos de recogida. Finalmente, se evaluaron diferencias intergrupales por género mediante la prueba $U$ de Mann-Whitney para dos muestras independientes. Para los análisis estadísticos fue utilizado el programa IBM-SPSS (versión 23.0). En cada caso, el nivel de significancia se estableció en 0,05.

\section{Análisis de datos cualitativos}

Los datos fueron analizados por un investigador ajeno al proceso de recogida de información. En primer lugar, se llevó a cabo una fase de codificación en la cual cada comentario vino asociado, al menos, a un color y una abreviatura. Tras un proceso de saturación, se obtuvieron las siguientes categorías: (a) responsabilidad, (b) relación entre iguales, (c) implicación y (d) neutra. Los comentarios fueron agrupados en función de dicha categorización, manteniendo su estructura temporal. Un mapeo posterior reveló fuertes conexiones entre las categorías responsabilidad e implicación, por lo que fueron reagrupadas derivando en una de las categorías finales. A efectos de consolidar la validez de los datos recabados se asumieron estrategias de triangulación temporal (Aguilar, y Barroso, 2015).

\section{RESULTADOS}




\section{Resultados cuantitativos}

La tabla 1 recoge los estadísticos descriptivos de cada una de las variables analizadas. No se reportan diferencias significativas en la dimensión de responsabilidad personal, en ninguna de las administraciones. Respecto a la responsabilidad social se observa un aumento significativo $(p<0,05)$ en varones y un descenso significativo en mujeres del GE-1 tras asumir roles fijos. En la variable comportamiento intercultural se produce la mayor cantidad de cambios significativos, encontrando efectos en ambos grupos experimentales y en todas las administraciones. Se observan aumentos significativos en la relación, ayuda y recompensa en varones de ambos grupos experimentales tras experimentar roles fijos, siendo estos efectos parcialmente extensibles a la muestra total. La dinámica rotativa provoca aumentos significativos en la recompensa en mujeres del GE-1 y varones del GE-2, así como en la relación en estudiantes del GE-1. Se halla un aumento significativo de la dimensión irresponsabilidad en mujeres y en la muestra total del GE-2. Finalmente, se evidencia un aumento de la REP en varones y en la $n$ total tras haber empleado roles fijos. La dinámica rotativa provoca un aumento significativo de la REP en el GE-2.

Tabla 1. Estadísticos descriptivos de las variables analizadas para la muestra total y género 
Rev.int.med.cienc.act.fís.deporte - vol. 20 - número 80 - ISSN: 1577-0354

\begin{tabular}{|c|c|c|c|c|c|c|c|c|c|c|c|c|}
\hline & \multicolumn{6}{|c|}{ GE-1 (Rotan - Fijos) } & \multicolumn{6}{|c|}{ GE-2 (Fijos - Rotan) } \\
\hline & \multicolumn{2}{|c|}{ PreT } & \multicolumn{2}{|c|}{ Post1 } & \multicolumn{2}{|c|}{ Post2 } & \multicolumn{2}{|c|}{ PreT } & \multicolumn{2}{|c|}{ Post1 } & \multicolumn{2}{|c|}{ Post2 } \\
\hline & $M$ & $D T$ & $M$ & $D T$ & $M$ & $D T$ & $M$ & $D T$ & $M$ & $D T$ & $M$ & $D T$ \\
\hline \multicolumn{13}{|c|}{ Responsabilidad Social } \\
\hline $\begin{array}{l}\text { Varone } \\
\mathrm{s}\end{array}$ & $4,66^{a b}$ & $\begin{array}{c}1,0 \\
7\end{array}$ & $4,44^{\mathrm{a}}$ & $\begin{array}{c}1,2 \\
2\end{array}$ & $4,70^{\mathrm{b}}$ & $\begin{array}{c}1,0 \\
4\end{array}$ & 4,73 & $\begin{array}{c}1,0 \\
5\end{array}$ & 5,08 & $\begin{array}{c}0,8 \\
5\end{array}$ & 5,15 & $\begin{array}{c}0,9 \\
2\end{array}$ \\
\hline Mujere & $\underset{\mathrm{a}}{5,34}$ & $\begin{array}{c}0,5 \\
7\end{array}$ & $\underset{b}{5,12^{a}}$ & $\begin{array}{c}0,8 \\
3\end{array}$ & $5,09^{b}$ & $\begin{array}{c}0,6 \\
8\end{array}$ & 5,32 & $\begin{array}{c}0,6 \\
9\end{array}$ & 5,31 & $\begin{array}{c}0,7 \\
0\end{array}$ & 5,14 & $\begin{array}{c}0,8 \\
6\end{array}$ \\
\hline \multicolumn{13}{|c|}{ Relación } \\
\hline $\mathrm{N}$ total & $\underset{\mathrm{a}}{4,09}$ & $\begin{array}{c}0,8 \\
6\end{array}$ & $4,28^{b}$ & $\begin{array}{c}0,8 \\
8\end{array}$ & $4,27^{b}$ & $\begin{array}{c}0,8 \\
8\end{array}$ & $4,08^{a}$ & $\begin{array}{c}1,0 \\
6\end{array}$ & $4,44^{b}$ & $\begin{array}{c}0,8 \\
0\end{array}$ & $\underset{b}{4,33^{a}}$ & $\begin{array}{c}0,8 \\
5\end{array}$ \\
\hline $\mathrm{s}$ & $\underset{\mathrm{a}}{3,90}$ & $\begin{array}{c}0,8 \\
9\end{array}$ & $\underset{b}{4,11^{a}}$ & $\begin{array}{c}0,9 \\
0\end{array}$ & $4,19^{b}$ & $\begin{array}{c}0,8 \\
1\end{array}$ & 3,89 & $\begin{array}{c}1,1 \\
1\end{array}$ & 4,36 & $\begin{array}{c}0,9 \\
1\end{array}$ & 4,30 & $\begin{array}{c}0,7 \\
5\end{array}$ \\
\hline \multicolumn{13}{|c|}{ Irresponsabilidad } \\
\hline $\mathrm{N}$ total & $\begin{array}{c}1,3 \\
6\end{array}$ & $\begin{array}{c}0,5 \\
5\end{array}$ & 1,32 & $\begin{array}{c}0,5 \\
2\end{array}$ & 1,40 & $\begin{array}{c}0,5 \\
8\end{array}$ & $\underset{b}{1,67^{a}}$ & $\begin{array}{c}0,9 \\
2\end{array}$ & $1,62^{\mathrm{a}}$ & $\begin{array}{c}1,1 \\
3\end{array}$ & $2,15^{\mathrm{b}}$ & $\begin{array}{c}1,2 \\
3\end{array}$ \\
\hline $\begin{array}{l}\text { Mujere } \\
\mathrm{s}\end{array}$ & $\begin{array}{c}1,2 \\
3\end{array}$ & $\begin{array}{c}0,5 \\
1\end{array}$ & 1,33 & $\begin{array}{c}0,6 \\
7\end{array}$ & 1,33 & $\begin{array}{c}0,5 \\
0\end{array}$ & $\underset{b}{1,38^{a}}$ & $\begin{array}{c}0,6 \\
5\end{array}$ & $1,15^{\mathrm{a}}$ & $\begin{array}{c}0,6 \\
0\end{array}$ & $1,91^{b}$ & $\begin{array}{c}1,0 \\
4\end{array}$ \\
\hline \multicolumn{13}{|c|}{ Recompensa } \\
\hline $\mathrm{N}$ total & $\underset{a}{3,84}$ & $\begin{array}{c}1,0 \\
3\end{array}$ & $4,05^{b}$ & $\begin{array}{c}1,0 \\
1\end{array}$ & $4,05^{b}$ & $\begin{array}{c}1,0 \\
0\end{array}$ & 4,08 & $\begin{array}{c}1,1 \\
4\end{array}$ & 4,40 & $\begin{array}{c}0,9 \\
0\end{array}$ & 4,29 & $\begin{array}{c}0,8 \\
9\end{array}$ \\
\hline Varone & $\underset{\mathrm{a}}{3,72}$ & $\begin{array}{c}1,1 \\
1\end{array}$ & $\underset{b}{3,85^{a}}$ & $\begin{array}{c}0,9 \\
4\end{array}$ & $4,02^{b}$ & $\begin{array}{c}0,8 \\
9\end{array}$ & $3,77^{a}$ & $\begin{array}{c}1,2 \\
6\end{array}$ & $4,32^{b}$ & $\begin{array}{c}1,0 \\
4\end{array}$ & $4,37^{b}$ & $\begin{array}{c}0,7 \\
9\end{array}$ \\
\hline $\begin{array}{l}\text { Mujere } \\
\text { s }\end{array}$ & $\begin{array}{c}3,95 \\
a\end{array}$ & $\begin{array}{c}0,9 \\
6\end{array}$ & $4,25^{b}$ & $\begin{array}{c}1,0 \\
7\end{array}$ & $\underset{b}{4,09^{a}}$ & $\begin{array}{c}1,1 \\
2\end{array}$ & 4,51 & $\begin{array}{c}0,8 \\
1\end{array}$ & 4,55 & $\begin{array}{c}0,6 \\
3\end{array}$ & 4,17 & $\begin{array}{c}1,0 \\
3\end{array}$ \\
\hline \multicolumn{13}{|l|}{ Ayuda } \\
\hline $\mathrm{N}$ total & 3,93 & $\begin{array}{c}1,0 \\
7\end{array}$ & 4,01 & $\begin{array}{c}1,0 \\
7\end{array}$ & 4,08 & $\begin{array}{c}0,9 \\
1\end{array}$ & $4,19^{a}$ & $\begin{array}{c}0,9 \\
2\end{array}$ & $4,46^{b}$ & $\begin{array}{c}0,7 \\
7\end{array}$ & $\underset{b}{4,22^{a}}$ & $\begin{array}{c}0,9 \\
6\end{array}$ \\
\hline $\begin{array}{l}\text { Varone } \\
\text { s }\end{array}$ & $\underset{\mathrm{a}}{3,81}$ & $\begin{array}{c}1,0 \\
5\end{array}$ & $\underset{b}{3,86^{a}}$ & $\begin{array}{c}1,0 \\
6\end{array}$ & $4,00^{b}$ & $\begin{array}{c}0,9 \\
1\end{array}$ & $3,91^{a}$ & $\begin{array}{c}0,9 \\
3\end{array}$ & $4,33^{b}$ & $\begin{array}{c}0,8 \\
3\end{array}$ & $\underset{b}{4,25^{a}}$ & $\begin{array}{c}0,8 \\
3\end{array}$ \\
\hline \multicolumn{13}{|l|}{ REP } \\
\hline $\mathrm{N}$ total & $\begin{array}{c}3,43 \\
a\end{array}$ & $\begin{array}{c}0,6 \\
1\end{array}$ & $\underset{b}{3,42^{a}}$ & $\begin{array}{c}0,6 \\
4\end{array}$ & $3,52^{b}$ & $\begin{array}{c}0,6 \\
4\end{array}$ & $3,37^{a}$ & $\begin{array}{c}0,7 \\
6\end{array}$ & $3,62^{b}$ & $\begin{array}{c}0,6 \\
5\end{array}$ & $3,52^{a}$ & $\begin{array}{c}0,5 \\
9\end{array}$ \\
\hline $\begin{array}{l}\text { Varone } \\
\text { s }\end{array}$ & $\underset{\mathrm{a}}{3,34}$ & $\begin{array}{c}0,5 \\
6\end{array}$ & $3,24^{a}$ & $\begin{array}{c}0,6 \\
8\end{array}$ & $3,41^{b}$ & $\begin{array}{c}0,6 \\
1\end{array}$ & $3,23^{a}$ & $\begin{array}{c}0,8 \\
1\end{array}$ & $\underset{b}{3,52^{a}}$ & $\begin{array}{c}0,6 \\
8\end{array}$ & $3,49^{b}$ & $\begin{array}{c}0,5 \\
5\end{array}$ \\
\hline
\end{tabular}

Nota: En cada fila, las medias con diferentes superíndices difieren al menos a un nivel de $p<0,05$. Para simplificar, se han omitido análisis cuando no emergieron cambios significativos.

A continuación, la tabla 2 presenta los resultados relativos a la prueba de rangos de Wilcoxon para cada una de las variables analizadas, establecidos según género y grupo experimental de pertenencia. 
Tabla 2. Puntuaciones $Z$ y significación asintótica bilateral (Sig. A. Bil.) de la prueba de rangos de Wilcoxon de las variables del estudio

\begin{tabular}{|c|c|c|c|c|c|c|}
\hline & \multicolumn{3}{|c|}{ GE-1 (Rotan - Fijos) } & \multicolumn{3}{|c|}{ GE-2 (Fijos - Rotan) } \\
\hline & $\begin{array}{l}\text { Post1 - } \\
\text { PreT }\end{array}$ & $\begin{array}{l}\text { Post2 - } \\
\text { PreT }\end{array}$ & $\begin{array}{l}\text { Post2 - } \\
\text { Post1 }\end{array}$ & $\begin{array}{l}\text { Post1 - } \\
\text { PreT }\end{array}$ & $\begin{array}{c}\text { Post2 - } \\
\text { PreT }\end{array}$ & $\begin{array}{l}\text { Post2 - } \\
\text { Post1 }\end{array}$ \\
\hline \multicolumn{7}{|c|}{ Responsabilidad Social } \\
\hline Varón Z & $-0,482^{b}$ & $-1,387^{b}$ & $-2,045^{b}$ & $-1,000^{b}$ & $-1,746^{b}$ & $-1,170^{b}$ \\
\hline Sig. asintót. (bilateral) & 0,630 & 0,166 & 0,041 & 0,317 & 0,081 & 0,242 \\
\hline Mujer Z & $-1,571^{b}$ & $-2,123^{b}$ & $-0,066^{b}$ & $-0,281^{b}$ & $-1,111^{b}$ & $-0,421^{b}$ \\
\hline Sig. asintót. (bilateral) & 0,116 & 0,034 & 0,948 & 0,779 & 0,267 & 0,674 \\
\hline \multicolumn{7}{|l|}{ Relación } \\
\hline $\mathrm{N}$ total $\mathrm{Z}$ & $-2,142^{b}$ & $-2,104^{b}$ &,$- 075^{b}$ & $-2,007^{b}$ & $-1,392^{b}$ & $-0,368^{b}$ \\
\hline Sig. asintót. (bilateral) & 0,032 & 0,035 & 0,940 & 0,045 & 0,164 & 0,713 \\
\hline Varón Z & $-1,373^{b}$ & $-2,469^{b}$ & $-0,625^{b}$ & $-1,852^{b}$ & $-1,597^{b}$ & $-0,213^{b}$ \\
\hline Sig. asintót. (bilateral) & 0,170 & 0,014 & 0,532 & 0,064 & 0,110 & 0,832 \\
\hline \multicolumn{7}{|l|}{ Irresponsabilidad } \\
\hline $\mathrm{N}$ total $\mathrm{Z}$ & $-1,363^{c}$ & $-0,473^{b}$ & $-0,886^{b}$ & $-1,401^{b}$ & $-1,361^{b}$ & $-2,219^{b}$ \\
\hline Sig. asintót. (bilateral) & 0,173 & 0,636 & 0,376 & 0,161 & 0,174 & 0,026 \\
\hline Mujer Z & $-0,071^{c}$ & $-0,898^{b}$ & $-0,362^{c}$ & $-1,633^{c}$ & $-1,206^{b}$ & $-2,536^{b}$ \\
\hline Sig. asintót. (bilateral) & 0,943 & 0,369 & 0,717 & 0,102 & 0,228 & 0,011 \\
\hline \multicolumn{7}{|l|}{ Recompensa } \\
\hline $\mathrm{N}$ total $\mathrm{Z}$ & $-2,449^{b}$ & $-2,127^{b}$ & $-0,446^{b}$ & $-1,659^{b}$ & $-0,810^{c}$ & $-0,525^{c}$ \\
\hline Sig. asintót. (bilateral) & 0,014 & 0,033 & 0,655 & 0,097 & 0,418 & 0,600 \\
\hline Varón Z & $-1,395^{c}$ & $-2,281^{b}$ & $-1,582^{b}$ & $-2,165^{b}$ & $-2,161^{c}$ & $-0,105^{c}$ \\
\hline Sig. asintót. (bilateral) & 0,163 & 0,023 & 0,114 & 0,030 & 0,031 & 0,916 \\
\hline Mujer Z & $-1,984^{b}$ & $-0,810^{c}$ & $-0,952^{b}$ & $-0,171^{c}$ & $-1,688^{b}$ & $-0,933^{b}$ \\
\hline Sig. asintót. (bilateral) & 0,047 & 0,418 & 0,341 & 0,865 & 0,091 & 0,351 \\
\hline \multicolumn{7}{|l|}{ Ayuda } \\
\hline $\mathrm{N}$ total $\mathrm{Z}$ & $-1,093^{b}$ & $-1,696^{b}$ & $-0,540^{b}$ & $-2,511^{b}$ & $-0,049^{c}$ & $-1,077^{c}$ \\
\hline Sig. asintót. (bilateral) & 0,274 & 0,090 & 0,589 & 0,012 & 0,961 & 0,281 \\
\hline Varón Z & $-0,774^{c}$ & $-2,072^{b}$ & $-0,865^{b}$ & $-2,777^{b}$ & $-1,164^{b}$ & $-0,029^{c}$ \\
\hline Sig. asintót. (bilateral) & 0,439 & 0,038 & 0,387 & 0,005 & 0,244 & 0,977 \\
\hline \multicolumn{7}{|l|}{ REP } \\
\hline $\mathrm{N}$ total $\mathrm{Z}$ & $-0,089^{b}$ & $-2,184^{b}$ & $-1,827^{b}$ & $-2,426^{b}$ & $-1,452^{b}$ & $-0,297^{b}$ \\
\hline Sig. asintót. (bilateral) & 0,929 & 0,029 & 0,068 & 0,015 & 0,147 & 0,767 \\
\hline Varón Z & $-0,896^{b}$ & $-2,491^{b}$ & $-1,996^{b}$ & $-1,648^{b}$ & $-2,024^{b}$ & $-0,229^{b}$ \\
\hline Sig. asintót. (bilateral) & 0,370 & 0,013 & 0,046 & 0,099 & 0,043 & 0,819 \\
\hline
\end{tabular}

Nota: En cada fila, las medias con diferentes superíndices difieren al menos a un nivel de $p$ $<0,05$. Se han omitido análisis cuando no emergieron cambios significativos.

Los resultados de la prueba $U$ de Mann-Whitney reflejan diferencias significativas en la distribución respecto al género, en las variables responsabilidad social (PreT y Post1) y personal (PreT) del GE-1, así como en las variables recompensa y ayuda (PreT) e irresponsabilidad y REP (Post1) del GE-2. 
Resultados cualitativos

A continuación, se presentan los resultados obtenidos de los datos cualitativos, a partir de las categorías emergentes: (I) percepción de responsabilidad e implicación, (II) relación entre iguales, relaciones colaborativas y conductas disruptivas.

\section{Percepción de responsabilidad e implicación}

En cuanto a la dinámica de roles asumida, la docente reconoció ventajas tanto para quienes realizaban roles fijos ("Más oportunidad de desarrollarse en la responsabilidad elegida", E1) como rotativos ("Si un rol no se le da bien, pues puede desempeñar otros", E1) advirtiendo de la dificultad que podría suponer su aprendizaje en alumnado sin experiencia con Educación Deportiva. Según los comentarios docentes, el nivel de responsabilidad aumentó "considerablemente" (E2) una vez concluida la primera intervención. Afirmó que en el grupo con roles rotativos, estos requerían de mayor tiempo para ser ejecutados, si bien no observó diferencias de consideración en su desempeño ("Tardaban un poquito más, pero lo realizaban también de forma correcta", E2). Esta circunstancia fue asociada al trabajo docente previo, al uso de tarjetas de rotación y a la percepción de una mayor empatía intragrupal.

El aumento en la responsabilidad fue igualmente destacado por la profesora tras finalizar la segunda intervención ("El hecho de que hayan estado trabajando juntos, pues yo creo que el nivel [de responsabilidad] ha aumentado", E3). En términos de género, tal y como aventuró inicialmente, no percibió diferencias destacables, aunque sí aseguró que, en estudiantes de sexo femenino, la responsabilidad aumentó considerablemente durante la fase de competición formal, siendo reseñable la manera en la que "tiraban de los varones" (E3). Respecto a los roles, la docente valoró el buen hacer de ambos grupos. Observó que, en el grupo con rotación, los cambios se realizaban correctamente ("Sabían perfectamente lo que les tocaba y lo que tenían que hacer", E3). El acceso a nuevas funciones (en su primera experiencia solo habían ejercido un rol) redundó en una mayor implicación. No afeaban la admisión de roles considerados como "menos deseables" sino que, como indicó la profesora, "si les gustaba un rol específico, sabían que ya llegaría" (E3). La profesora destacó un clima de trabajo más sosegado en aquellos que ejercieron roles fijos. Desde su perspectiva, esto se debió al hecho de tener bien automatizados cada uno de los roles.

\section{Relación entre iguales, relaciones colaborativas y conductas disruptivas}

Según la perspectiva docente, la responsabilidad y el establecimiento de normas repercutieron positivamente en las relaciones entre iguales ("Al establecer las normas, los roles y otras características del modelo, las relaciones han mejorado", E2). Inicialmente, estaban marcadas por la existencia de discusiones y conflictos asiduos ("Las primeras sesiones, observaba falta de responsabilidad, no tenían respeto hacia las normas, discutían mucho", E3), sin existir apenas relaciones colaborativas. Se mostró algo más ambigua respecto a la fase de competición ("Ahí podría haber conflictos", E1). Aun considerando que los 
conflictos no estarían marcados por las características culturales del alumnado, entendió que una menor implicación podría derivar en mayores situaciones conflictivas.

Tras finalizar la primera unidad, la docente apreció una "evidente" mejora de las relaciones sociales (E2), con especial incidencia en estudiantes de origen inmigrante, reflejado en una mayor integración y creación de nuevos vínculos. A nivel intragrupal, la afiliación fue manifiesta, apuntando cómo la disconformidad inicial respecto a los agrupamientos se tornó en aceptación en ambos grupos ("Han estado muy bien en toda la experiencia y, al final, algunos incluso me agradecían que, (...) independientemente de que estuvieran con sus amigos, habían trabajado y se lo estaban pasando bien", E2). Según la docente, existieron diferencias en términos de colaboración inter e intragrupal. Aseguró que en situaciones en las que un igual tenía dificultades en el ejercicio de su función, un compañero/a, valiéndose de su conocimiento previo del rol, le prestaba ayuda. Así lo ejemplificó, en la última entrevista, con el rol de capitán: "Otros que habían sido capitán antes, si veían que un compañero tenía problemas, le ayudaban". Estas ayudas surgieron independientemente del género y de la nacionalidad del alumnado.

Las relaciones de colaboración se mantuvieron durante la segunda unidad. Sin embargo, la docente destacó que ciertas acciones sumamente apreciadas en la unidad anterior (por ejemplo, feedback intragrupal al ejercer las responsabilidades), en esta ocasión "no eran tan necesarias" (E3) al observar un trabajo individual más fluido. Los conflictos disminuyeron, siendo menos presentes que en la primera unidad. Así fue descrito por la profesora: "Los conflictos se han eliminado y hemos pasado de, prácticamente, no poder jugar, porque nadie respetaba una norma, a no solamente respetar las normas, sino que los conflictos, si se dan, se solucionan" (E3). Varios argumentos emergieron de los comentarios docentes: (a) el asentamiento del fair play, (b) la presión intragrupal y (c) la figura del mediador ("Los mediadores se ocupaban de que esto no ocurriera (...). En el momento que sucedía algo, que puede pasar, se eliminaba y el grupo continuaba", E3). El rol de mediador fue decisivo durante la fase de competición donde, según la docente, se concentraron la mayoría de los conflictos. Las mejoras en los conflictos aparecieron también en términos interculturales. La docente destacó el caso de un alumno de etnia gitana cuyo comportamiento, más negativo a priori, mejoró con la segunda experiencia.

\section{DISCUSIÓN Y CONCLUSIONES}

El presente estudio ha examinado los efectos de dos intervenciones con Educación Deportiva, distinguidas por la dinámica de roles asumida (fija o rotativa), sobre tres variables socio-afectivas en adolescentes de un centro de enseñanza en un contexto socialmente vulnerable. Se estableció como hipótesis de partida que la asunción de roles rotativos provocaría mejoras significativas tanto en la responsabilidad personal como social, siendo estas superiores a las suscitadas por los roles fijos. En oposición a esta primera hipótesis, los resultados demuestran efectos positivos significativos $(p<0,05)$ de los roles fijos sobre la responsabilidad social en varones del GE-1, sin existir incidencia significativa de la rotación. Estos resultados difieren de aquellos revelados por 
Menéndez-Santurio y Fernández-Río (2016; 2017), así como de lo informado por Fernández-Río et al. (2017) quienes en sendas experiencias con el modelo (los primeros a través de una propuesta híbrida), encontraron mejoras en la responsabilidad social de adolescentes españoles, sirviéndose de roles rotativos. Al igual que en este estudio, en las experiencias citadas el alumnado afrontaba por primera vez el aprendizaje con Educación Deportiva.

Inesperadamente, la asunción de roles fijos provocó un descenso significativo en la responsabilidad social en las mujeres del GE-1 tras la segunda experiencia, divergiendo de lo observado por la docente quien destacó mejoras en el nivel de responsabilidad desarrollado por las estudiantes. Este hecho hace presagiar consecuencias negativas derivadas de un efecto sumativo de la intervención, pudiendo estar supeditado a la naturaleza del contenido (deportes de invasión) y la consecuente asunción de un rol jugado menos protagónico por parte de las estudiantes. Un estudio reciente ha evidenciado relaciones positivas entre los niveles de responsabilidad social y el nivel de actividad física practicado en estudiantes adolescentes (Gómez-Mármol, Sánchez-Alcaraz, De la Cruz, Valero, y González-Víllora, 2017). Futuras intervenciones deberían explorar los efectos de la Educación Deportiva y sus dinámicas de roles, sobre la responsabilidad del alumnado y los niveles de actividad física, considerando la inclusión de contenidos de diferente naturaleza. Por su parte, en el grupo donde los estudiantes rotaron roles después de haber asumido roles fijos (GE-2) no se observaron cambios de significatividad. Considerando las características del contexto y el escenario de partida, el hecho de que la dinámica rotativa no promoviese cambios significativos en ninguno de los grupos experimentales, debe ser asumido como positivo. Tampoco emergieron diferencias significativas en la responsabilidad personal, en ambos grupos, si bien se observaron tendencias similares a las evidenciadas en la dimensión social (ligeras caídas al trabajar con roles rotativos seguidas de una recuperación al asumir roles fijos; descensos sostenidos en el tiempo en mujeres).

En contra de la segunda hipótesis, los resultados cuantitativos revelan un mayor impacto de la dinámica de roles fijos respecto a la rotativa, al provocar incrementos significativos en cada una de las dimensiones positivas del comportamiento intercultural en estudiantes de ambos grupos, con especial incidencia en varones. Los resultados coinciden parcialmente con lo advertido por Méndez-Giménez et al. (2017) quienes, en una experiencia con Educación Deportiva sobre mimo, y tras emplear roles fijos, revelaron aumentos significativos en la dimensión de ayuda en un grupo de preadolescentes. En el presente estudio, la inclusión del rol de mediador supuso una novedad respecto a lo realizado en aquel (Méndez-Giménez, et al. 2017), siendo decisiva tanto en la creación de relaciones colaborativas como en la disminución de conflictos. Autores como Farias (2017) subrayan la importancia del rol de mediador en el desarrollo de la responsabilidad social, siendo la ayuda un valor asociado a dicha dimensión.

La dinámica rotativa, si bien provocó efectos positivos (aumentos significativos en las dimensiones de relación y recompensa), de forma insospechada, promovió un aumento de la irresponsabilidad en mujeres del GE-2. Estos resultados caminan en la misma línea de aquellos encontrados en la 
responsabilidad social. Los efectos derivados de la interacción de dinámicas, en perjuicio de la rotativa, parecen desvelar la importancia del uso de roles fijos en alumnado sin experiencia con el modelo, haciendo extensible su conveniencia a estudiantes inicialmente apáticos respecto al contenido. El hecho de que las consecuencias más negativas se presenten en segundas experiencias, independientemente de la condición experimental, lleva a entrever secuelas en estudiantes de sexo femenino derivadas de un efecto prolongado del modelo. En términos de estatus social (Brock, Rovegno, y Oliver, 2009), cabe argüir que en las dinámicas rotativas el liderazgo va cambiando, pudiendo causar problemas de aceptación en determinados roles, siendo más sensible en contextos socialmente vulnerables. Futuras intervenciones podrán dar cuenta de estas circunstancias.

Fue igualmente hipotetizado que el grupo que trabajase a partir de roles rotativos demostraría mejoras significativas en la REP superiores a las provocadas por la dinámica fija. Los participantes, en ambos grupos, exhibieron aumentos significativos en dicha variable cuando fueron enseñados usando roles fijos, convergiendo así con los hallazgos de Puente-Maxera, Méndez-Giménez y Martínez de Ojeda (2018a) en estudiantes preadolescentes. Por su parte, la dinámica rotativa provocó aumentos significativos en la REP, si bien estos solo emergieron en varones del GE-2. Estos resultados, en conjunción con los anteriores, rechazan la tercera hipótesis planteada. El aumento en la REP hacia el contacto intercultural ha sido informado en un estudio previo con Educación Deportiva (Puente-Maxera et al., 2017) donde, tras comparar las dinámicas de roles, se observó una mayor incidencia de la rotativa. En sintonía con lo advertido por dichos autores, en el estudio actual se destaca, desde el plano cualitativo, la noción de afiliación como aspecto decisivo. Las mejoras derivadas de la rotación de roles en la segunda experiencia podrían ser interpretadas como consecuencias de un efecto reforzado de los roles fijos inicialmente asumidos. Se evidencia la necesidad de futuras intervenciones orientadas en esta línea.

Los hallazgos del estudio actual sugieren que, en contextos socialmente vulnerables, los roles fijos reportan beneficios a corto plazo. No obstante, será necesario el abordaje de estudios prolongados a efectos de dilucidar este aspecto. En términos interculturales, parecen desvelarse coincidencias con lo advertido por la literatura sobre el potencial del modelo de Educación Deportiva en la promoción de relaciones positivas entre iguales de diferentes antecedentes étnico-culturales, así como en el desarrollo de actitudes prosociales como la ayuda, el respeto y el reconocimiento mutuo. No obstante, resulta oportuno poner en tela de juicio una serie de limitaciones presentes en este estudio: (a) el diseño de investigación, carente de grupo control, (b) la concreción a un único centro de enseñanza y con cursos impartidos por una única docente y (c) el bajo tamaño muestral respecto a alumnado de origen inmigrante lleva a tratar con cautela las interpretaciones respecto a dicho sub-grupo.

Futuras investigaciones podrán reforzar tendencias, por ejemplo, aumentando el número de docentes en diferentes centros y en diferentes contextos (p.ej., alumnado con experiencia con el modelo; centros de estrato socioeconómico medio-alto; alumnado de primaria). Asimismo, es importante enfatizar que la intervención fue llevada a cabo a partir de dos deportes de misma naturaleza o 
categoría táctica, abogando por futuras intervenciones que consideren esta circunstancia. Será igualmente interesante analizar variables adicionales que vayan más allá de lo social (rendimiento de juego, motivación, etc.).

\section{REFERENCIAS BIBLIOGRÁFICAS}

Aguilar, S., y Barroso, J. (2015). La triangulación de datos como estrategia en investigación educativa. Píxel-Bit. Revista de Medios y Educación, 47, 7388. https://doi.org/10.12795/pixelbit.2015.147.05

Araújo, R., Mesquita, I., y Hastie, P.A. (2014). Review of the Status of Learning in Research on Sport Education: Future Research and Practice. Journal of Sports Science and Medicine, 13, 846-858.

Brock, S. J., Rovegno, I., y Oliver, K. (2009). The influence of student status on student interactions and experiences during a sport education unit. Physical Education and Sport Pedagogy, 14(4), 355-375. https://doi.org/10.1080/17408980802400494

Calderón, A., Martínez de Ojeda, D., y Hastie, P. A. (2013). Students and teachers' perception after practice with two pedagogical models in Physical Education. International Journal of Sport Science, 32(9), 137153. https://doi.org/10.5232/ricyde2013.03204

Casey, A., y Dyson, B. (2009). The implementation of models-based practice in physical education through action research. European Physical Education Review, 15(2), 175-199. https://doi.org/10.1177/1356336X09345222

Contreras, O. R., Gil, P., Cecchini, J. A., y García-López, L. M. (2007): Teoría de una educación física intercultural y realidad educativa en España. Revista Paradigma, 28(2), 7-47.

Escartí, A., Gutiérrez, M., y Pascual, C. (2011). Propiedades psicométricas de la versión española del Cuestionario de Responsabilidad Personal y Social en contextos de educación física. Revista de Psicología del Deporte, 20(1), 119-130.

Farias, C. (2017). Promoting equity and social responsibility within Sport Education. ACHPER Active and Healthy Journal, 24, 36-42.

Fernández-Río, J., Méndez-Giménez, A., y Méndez-Alonso, D. (2017). Efectos de dos formatos instructivos, Educación Deportiva e Instrucción Directa, en la respuesta psicológica de estudiantes de secundaria. SportTK, 6(2), 9-20.

García-López, L. M., y Gutiérrez, D. (2013). The effects of a sport education season on empathy and assertiveness. Physical Education and Sport Pedagogy, 14, 355-375. https://doi.org/10.1080/17408989.2013.780592

Gómez-Mármol, A., Sánchez-Alcaraz, B. J., De la Cruz, E., Valero, A., y González-Víllora, S. (2017). Personal and social responsibility development through sport participation in youth scholars. Journal of Physical Education and Sport, 17(2), 775 - 782. https://doi.org/10.7752/jpes.2017.02118

Grimminger-Seidensticker, E., y Möhwald, A. (2017). Intercultural education in physical education: results of a quasi-experimental intervention study with secondary school students. Physical Education and Sport Pedagogy, 22(5), 445-458. https://doi:10.1080/17408989.2016.1225030 
Hastie, P. A., y Casey, A. (2014). Fidelity in models-based practice research in sport pedagogy: A guide for future investigations. Journal of Teaching in Physical Education, 33(3), 422-431. https://doi.org/10.1123/itpe.20130141

Hastie, P. A., Martínez de Ojeda, D., y Calderón, A. (2011). A review of research on Sport Education: 2004 to the present. Physical Education and Sport Pedagogy, 16(2),

103-132. https://doi.org/10.1080/17408989.2010.535202

Hastie, P.A., Sinelnikov, O.A., y Guarino, A. J. (2009). The development of skill and tactical competencies during a season of badminton. European Journal of Sport Science, 9(3), 133-140. https://doi.org/10.1080/17461390802542564

Hastie, P. A., Ward, J. K., y Brock, S. J. (2017). Effect of graded competition on student opportunities for participation and success rates during a season of Sport Education. Physical Education and Sport Pedagogy, 22(3), 316327. https://doi.org/10.1080/17408989.2016.1203888

Haudenhuyse, R., Theeboom, M., y Nols, Z. (2012). Sports-based interventions for socially vulnerable youth: Towards well-defined interventions with easy-to-follow outcomes? International Review for the Sociology of Sport, O(0), 1-14. https://doi.org/10.1177/1012690212448002

Kellis, I., Vernadakis, N., Albanidis, E., Derri, V., y Kourtesses, T. (2010). The development of a student's behaviors' self-evaluation scale (SBSS) in multicultural physical education class settings. Educational Research and Review, 5(11), 637-645. https://doi.org/10.5897/ERR.9000336

Martínez de Ojeda, D., Méndez-Giménez, A., y Valverde, J. J, (2016). Efectos del modelo Educación Deportiva en el clima social del aula, la competencia percibida y la intención de ser físicamente activo: un estudio prolongado en primaria. Sport TK, 5(2), 153-165.

Méndez-Giménez, A., Fernández-Río, J., y Méndez-Alonso, D. (2015). x. Revista Internacional de Medicina y Ciencias de la Actividad Física y el Deporte, 15(59), 449-466. http://dx.doi.org/10.15366/rimcafd2015.59.004

Méndez-Giménez, A., Puente-Maxera, F., y Martínez de Ojeda, D. (2017). Efectos de una unidad didáctica de mimo basada en el modelo de Educación Deportiva sobre la interculturalidad. Sport TK, 6(2), 89-100.

Menéndez-Santurio, J. I., y Fernández-Río, J. (2016). Violencia, responsabilidad, amistad y necesidades psicológicas básicas: efectos de un programa de Educación Deportiva y Responsabilidad Personal y Social. Revista de Psicodidáctica, 21(2), 245-260. https://doi.org/10.1387/RevPsicodidact.15269

Menéndez-Santurio, J. I., y Fernández-Río, J. (2017). Teachers and Students' Perceptions of a Hybrid Sport Education and Teaching for Personal and Social Responsibility Learning Unit. Journal of Teaching in Physical Education, 36, 185 -196. https://doi.org/10.1123/itpe.2016-0077

Metzler, M. W. (2011). Instructional models for physical education (2ª ed.). Scottsdale, AZ: Holcomb Hathaway Publishing.

Nunnally, J. C. (1978). Psychometric theory. New York: McGraw-Hill.

Pastor-Vicedo, J. C., Contreras, O. R., Gil-Madrona, P., y Cuevas-Campos, R. (2016). Estereotipos y prejuicios hacia los inmigrantes en los futuros 
profesores de educación física. Cuadernos de Psicología del Deporte, 16(3), 123-136.

Puente-Maxera, F., Méndez-Giménez, A., y Martínez de Ojeda, D. (2018a). Efectos del modelo de Educación Deportiva sobre la interculturalidad: El papel del alumno-entrenador en estudiantes de primaria. Espiral. Cuadernos del Profesorado, 11(22), 1-14.

Puente-Maxera, F., Méndez-Giménez, A., y Martínez de Ojeda, D. (2018b). Modelo de Educación Deportiva y dinámica de roles. Efectos de una intervención sobre las variables motivacionales de estudiantes de primaria. Cultura, Ciencia y Deporte, 13(39), 281-290.

Puente-Maxera, F., Méndez-Giménez, A., y Martínez de Ojeda, D. (2017). Influencia de la rotación de roles en Educación Deportiva sobre la sensibilidad intercultural del alumnado de primaria. Revista Nacional e Internacional de Educación Inclusiva, 10(2), 165-180.

Rivas, M. (2002). Innovación educativa. Madrid: Síntesis.

Ruíz, J. R. (1996). Metodología de la investigación cualitativa. Bilbao: Universidad de Deusto.

Sanhueza, S. V., y Cardona, M. C. (2008). Escala de Sensibilidad Intercultural para Alumnado de Primaria y Secundaria (documento inédito). Alicante: Universidad de Alicante.

Serra, M., y Canciano, E. (2006). Las condiciones de enseñanza en contextos críticos. Buenos Aires: Ministerio de Educación.

Siedentop, D. (1994). Sport education: Quality PE through positive sport experiences. Champaign, IL: Human Kinetics.

Siedentop, D., Hastie, P. A., y van Der Mars, H. (2011). Complete Guide to Sport Education. ( $2^{\mathrm{a}}$ ed.). Champaign, IL: Human Kinetics.

Spittle, M., y Byrne, K. (2009). The influence of Sport Education on student motivation in physical education. Physical Education and Sport Pedagogy, 14(3), 253-266. https://doi.org/10.1080/17408980801995239

Subirats, H. J., Carmona, R. G., y Torruella, J. B. (2005). Análisis de los factores de exclusión social. Fundación BBVA. Recuperado de http://www.inau.gub.uy/biblioteca/exclusion social.pdf

Número de referencias totales / Total references: 37 (100\%) Número de citas propias de la revista / Journal's own references: $1(3 \%)$

Rev.int.med.cienc.act.fís.deporte - vol. 20 - número 80 - ISSN: 1577-0354 